\title{
Chronic pelvic pain caused by Herlyn-Werner-Wunderlich Syndrome: Case report and discussion
}

\author{
Fatma Zahra Affes ${ }^{1}$, Hatem Frikha ${ }^{2}$, Sana Menjli², Abir Karoui ${ }^{2}$, Sami Mahjoub ${ }^{1}$, \\ Mohamed Badis Chanoufi ${ }^{2}$, and Hassine Saber Abouda ${ }^{2}$ \\ ${ }^{1}$ Centre de Maternité et de Neonatologie \\ ${ }^{2}$ Affiliation not available
}

August 28, 2020

\section{Chronic pelvic pain caused by Herlyn-Werner-Wunderlich Syndrome: Case report and discus- sion}

Affes Fatma Zahra, Resident in obstetrics and gynaecology ${ }^{1}$, Frikha Hatem, Resident in obstetrics and gynae$\operatorname{cology}^{1}$, Menjli Sana, Physician assistant in obstetrics and gynaecology ${ }^{1}$, Karoui Abir, Physician assistant in obstetrics and gynaecology ${ }^{1}$, Mahjoub Sami, Professor in obstetrics and gynaecology ${ }^{1}$, Chanoufi Mohamed Badis, Professor in obstetrics and gynaecology ${ }^{1}$,Abouda Hassine Saber, Associate Professor in obstetrics and gynaecology ${ }^{1}$

Author's affiliations:

${ }^{1}$ Departement of Gynaecology $\ll \mathrm{C} \gg$ Centre de maternité et de néonatologie de Tunis

Name and contact details:

Affes Fatma Zahra

Email: fatmazahra310@gmail.com

Phone : +216 21444833

Address : Centre de maternité et de néonatologie de Tunis 1007 La Rabta Tunis, Tunisia.

Introduction:

HWW Syndrome also known as OHVIRA (obstructed hemivagina and ipsilateral renal agenisis) is a rare genitourinary anomaly that occurs in females. It is classified as class III Mullerian dygenisis.(1) This condition involves uterus didelphys and blind hemivagina associated with ipsilateral renal agnenisis. It represents $5 \%$ of total Mullerian dysgenisis(2). The onset of symptoms usually begins after the menarche. Abdominal and pelvic pain are the most commun symptoms. The diagnosis is difficult because of the infrequency of the syndrome and requires precise imaging to evaluate uterine cervical and vaginal morphology. Early detection and treatment of the syndrome can relieve the symptoms, improve the quality of life and prevent serious complications.(3) We present here a case of investigation and management this condition with pelvic pain in young female.

Presentation of case:

A 15 year-old female was referred to our university hospital with complaint of pain in the lower abdomen on the right side. She reported that she has the same chronic pain since her first menses at the age of twelve. She described her pain as mild in the first episodes then increasing gradually with each subsequent menstrual 
cycle. Her menstrual cycles were irregular with moderate bleeding. She haven't had any sexual intercourse before.

Physical examination revealed a tender lump in the pelvic region on the right side of midline. Secondary sex growth was normal but the vaginal examination was not done.

Ultrasound examination of the pelvis was first performed exhibiting uterus didelphys. The right uterine cavity and cervical canal showed a collection suggestive of hematometra and hematocervix, the vagina was also dilated containing hypoechoic collection. The left uterine cavity and cervix were normal with homogenous endometrium. Both ovaries were normal.

We performed an ultrasound of the abdomen searching urinary tract anomaly, and it revealed the agenesis of the right kidney with mild compensatory hypertrophy of the left kidney.

MRI of the abdomen and the pelvis confirmed the findings of the ultrasound examination: The right kidney was absent in the right renal fossa with mild compensatory hypertrophy of the left kidney. Normal left uterus with its own corpus and cervix, the right uterus cavity was stretched and contained hypodense material and was connected to a cystic mass by its cervical canal obstructing the vagina.

A diagnosis of uterus didelphys with right hematometra, hematocervix and hematocolpos with right renal agenesis suggestive of the Herlyn-Werner-Wanderlich syndrome was made.

Surgical intervention was planned. We first performed vaginoscopy under general anesthesia that showed a small vagina, normal cervix and uterus with one ostium. Septoplasty was prepared but was not possible because of the small size of the vagina, the septum was thick and profound. We decided to puncture the hematocolpos to evacuate the blood and relieve the pain. The puncture was difficult because of the thickness of the blood clots so infusion of saline serum was first done then the puncture was easier, and $300 \mathrm{cc}$ of blood was evacuated. The patient did well postoperatively and we decided then to inject medroxyprogesterone acetate every three months to prevent the accumulation of the blood in the right uterus and vagina. Further evaluation showed an asymptomatic patient, and ultrasound did not show any blood accumulation in the uterus or the blind vagina.

Discussion:

Herlyn Werner Wanderlich syndrome is a rare congenital defect in the development of genitourinary tract. It is characterized by uterus didelphys with obstructed hemivagina due to lateral non-fusion of mullerian duct with asymmetric obstruction. It is associated to renal agenesis ipsilateral to the obstructed vagina.(4)

This syndrome was described for the first time in 1971 by Herlyn and Werner. Then in 1976 renal aplasia with bicornuate uterus and simple vagina in the presence of an isolated hematocervix was described by Wunderlich.(5)

This kind of anomaly was classified to two types: type I a completely obstructed vagina and type II an incompletely obstructed vagina.(3)

The clinical presentation depends on the type of obstruction, it usually consists on severe dysmenorrhea starting after the menarche. The pain typically increases due to the volume of the hematometrocolpos when the vagina is completely obstructed however incomplete obstruction may be the origin of intermittent and mild symptoms. (6)

Besides hematometra, complete obstruction causes hematosalpinx as well as bleeding in the periadnexal and peritoneal space. Endometriosis may also occur as a consequence of the blood reflux in the peritoneal cavity and progress casing pelvic adhesions and infertility.(3)

Few cases of acute abdominal pain, abnormal vaginal discharge or acute retention of urine has been reported.(7) Rarely, spontaneous perforation of the obstructed vagina may occur leading to pyocolpos and pelvic inflammatory disease and also septic shock.(8) 
The diagnosis of the Mullarian duct anomalies can be done using HSG, US, 3D US and MRI.(9) Conventional ultrasonography can detect the hematocolpos and help to identify the type of the Mullarian duct anomalies because it displays the features of the external uterine contour.(10) MRI is the gold standard for diagnosis with higher sensitivity in detecting the uterine morphology and the continuity of the vagina, thickness and localization of the vaginal septum.(7) However, the agreement between the 3D US and MRI has been also shown in several studies in the evaluation of this type of anomalies.(11)

HSG was used before the development of MRI and US, its ability is limited because it is unable to display the external contour of the uterus. (9)

Laparoscopy is not necessary nowadays for the diagnosis thinks to the advanced imaging technologies and it is reserved only if the diagnosis isn't clear after imaging. (12)

Currently, the main treatment of the OHVIRA syndrome is full resection of the vaginal septum in order to achieve the continuity of vagina.(13) Vaginoplasty can be performed in single stage where the resection of the vaginal septum is complete or in two stages: draining the hematocolpos first then resection of the septum.(8) Infection, anatomic distortion and incomplete previous resection were the main indication of two stages vaginoplasty.(14) Laparoscopy doesn't seem to be required for the surgical management in most cases.(14)

However, Fauchin and al reported a case of hemiuterus with absent cervix where the vaginoplasty was not possible, the treatment was a laparoscopic hemi-hysterectomy with salpingectomy.(15) Rarely, hemi hysterectomy may be also considered in case of recurrent stenosis.(7)

Hysteroscopic resection of the septum under transabdominal guidance has also been reported in order to preserve the integrity of the hymen.(16)

When postponement of surgery is needed for young adolescents, amenorrhea can be maintained by the gonadotrophin-releasing hormone analogues.(13)

Conclusion:

OHVIRA syndrome is the most common obstructive Mullarian duct anomaly diagnosed after menarche in young adolescent. It is responsible of cyclic pain. It is usually treated with vaginoplasty and drainage of the hematocolpos. In case of recurrent stenosis or difficult septum resection, unilateral hysterectomy is the treatment of choice.

Acknowledgements:

We would like to express our appreciation to our department team for their valuable assistance.

Disclosure of interest:

No conflicts of interest.

Contribution to authorship:

Dr Affes Fatma Zahra: Initial diagnostics end treatment of the patient and case report manuscript.

Dr Frikha Hatem: Initial diagnostics and treatment of the patient and case report manuscript.

Dr Menjli Sana: Initial diagnostics and treatment of the patient.

Dr Karoui Abir: Initial diagnostics and treatment of the patient.

Dr Chanoufi Mohamed Badis: Initial diagnostics and treatment of the patient.

Dr Mahjoub Sami: Intitial diagnostics and treatment of the patient.

Dr Abouda Hassine Saber: Initial diagnostics and treatment of the patient.

Details of Ethics Approval 
Not applicable

Funding:

We don't have any external funding.

Consent for publication:

A consent was signed by the parents of the patient.

References

1. Epelman M, Dinan D, Gee MS, Servaes S, Lee EY, Darge K. Müllerian Duct and Related Anomalies in Children and Adolescents. Magn Reson Imaging Clin N Am. 1 nov 2013;21(4):773-89.

2. Khaladkar SM, Kamal V, Kamal A, Kondapavuluri SK. The Herlyn-Werner-Wunderlich Syndrome - A Case Report with Radiological Review. Pol J Radiol. 2016;81:395-400.

3. Zhu L, Chen N, Tong J-L, Wang W, Zhang L, Lang J-H. New Classification of Herlyn-Werner-Wunderlich Syndrome. Chin Med J (Engl). 20 janv 2015;128(2):222-225.

4. Ilyas M, Khan I, Saldanha CL. Herlyn-Werner-Wunderlich syndrome - a rare genitourinary anomaly in females: a series of four cases. Pol J Radiol. 2018;83:306-10.

5. Wunderlich M. [Unusual form of genital malformation with aplasia of the right kidney]. Zentralbl Gynakol. 1976;98(9):559-62.

6. Karaca L, Pirimoglu B, Bayraktutan U, Ogul H, Oral A, Kantarci M. Herlyn-Werner-Wunderlich Syndrome: A Very Rare Urogenital Anomaly in a Teenage Girl. J Emerg Med. 1 mars 2015;48(3):e73-5.

7. Gungor Ugurlucan F, Bastu E, Gulsen G, Kurek Eken M, Akhan SE. OHVIRA syndrome presenting with acute abdomen: a case report and review of the literature. Clin Imaging. juin 2014;38(3):357-9.

8. Ugurlucan FG, Dural O, Yasa C, Kirpinar G, Akhan SE. Diagnosis, management, and outcome of obstructed hemivagina and ipsilateral renal agenesis (OHVIRA syndrome): Is there a correlation between MRI findings and outcome? Clin Imaging. 1 fevr 2020;59(2):172-8.

9. Coleman, A.D., Arbuckle, J.L. Advanced Imaging for the Diagnosis and Treatment of Coexistent Renal and Mullerian Abnormalities. Curr Urol Rep 19, 89 (2018).

10. Troiano RN, McCarthy SM. Mullerian Duct Anomalies: Imaging and Clinical Issues. Radiology. 1 oct 2004;233(1):19-34.

11. Berger A, Batzer F, Lev-Toaff A, Berry-Roberts C. Diagnostic Imaging Modalities for Mullerian Anomalies: The Case for a New Gold Standard. J Minim Invasive Gynecol. 1 mai 2014;21(3):335-45.

12. Widyakusuma LS, Lisnawati Y, Pudyastuti S, Haloho AH. A rare case of pelvic pain caused by HerlynWerner-Wunderlich Syndrome in an adult: A case report. Int J Surg Case Rep. 1 janv 2018;49:106-9.

13. Tong J, Zhu L, Lang J. Clinical characteristics of 70 patients with Herlyn-Werner-Wunderlich syndrome. Int J Gynaecol Obstet Off Organ Int Fed Gynaecol Obstet. mai 2013;121(2):173-5.

14. Smith NA, Laufer MR. Obstructed hemivagina and ipsilateral renal anomaly (OHVIRA) syndrome: management and follow-up. Fertil Steril. 1 avr 2007;87(4):918-22.

15. Girardi Fachin C, Aleixes Sampaio Rocha JL, Atuati Maltoni A, das Chagas Lima RL, Arias Zendim V, Agulham MA, et al. Herlyn-Werner-Wunderlich syndrome: Diagnosis and treatment of an atypical case and review of literature. Int J Surg Case Rep. 1 janv 2019;63:129-34.

16. Kim TE, Lee GH, Choi YM, Jee BC, Ku SY, Suh CS, et al. Hysteroscopic resection of the vaginal septum in uterus didelphys with obstructed hemivagina: a case report. J Korean Med Sci. aout 2007;22(4):766-9. 

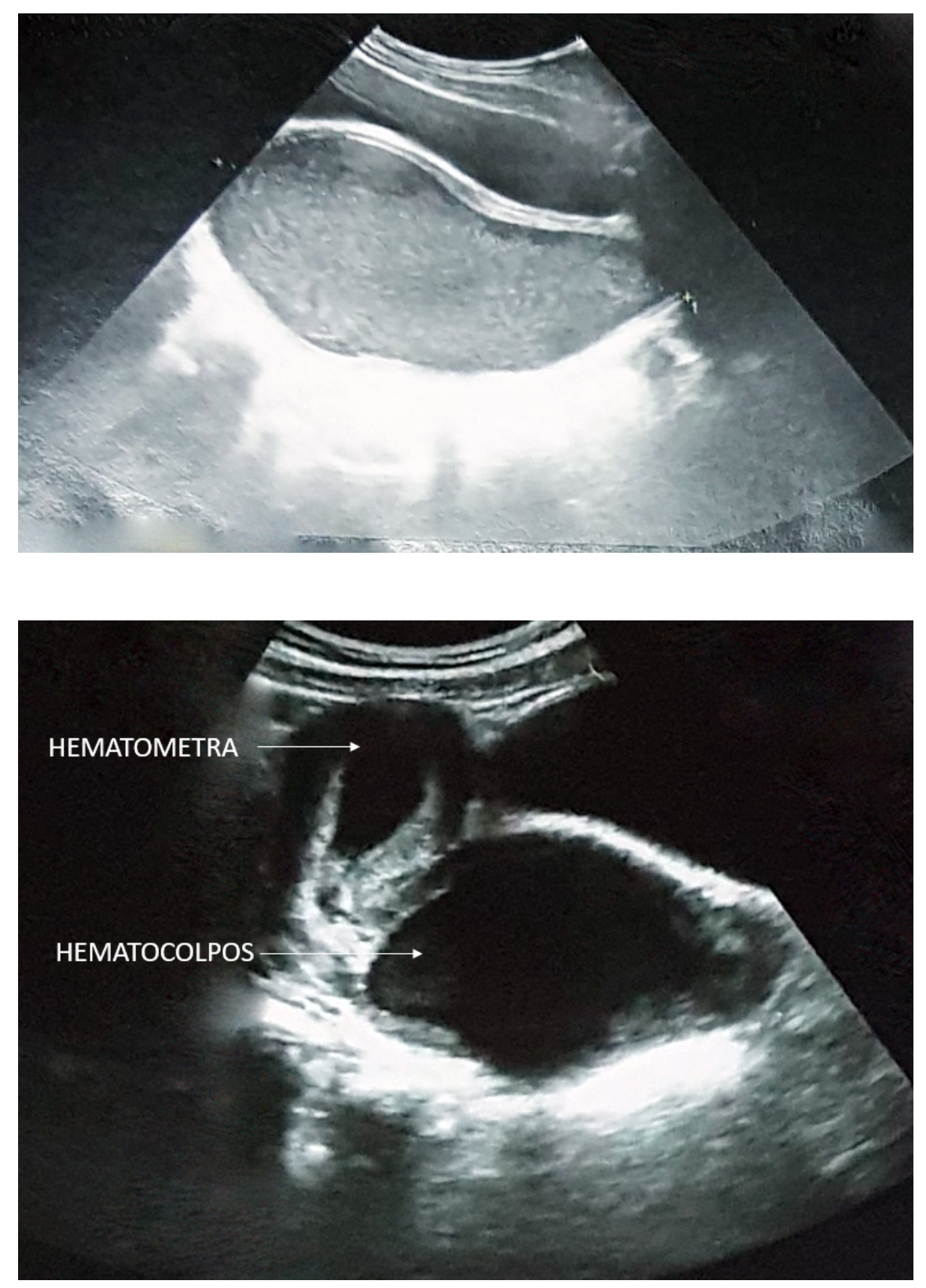


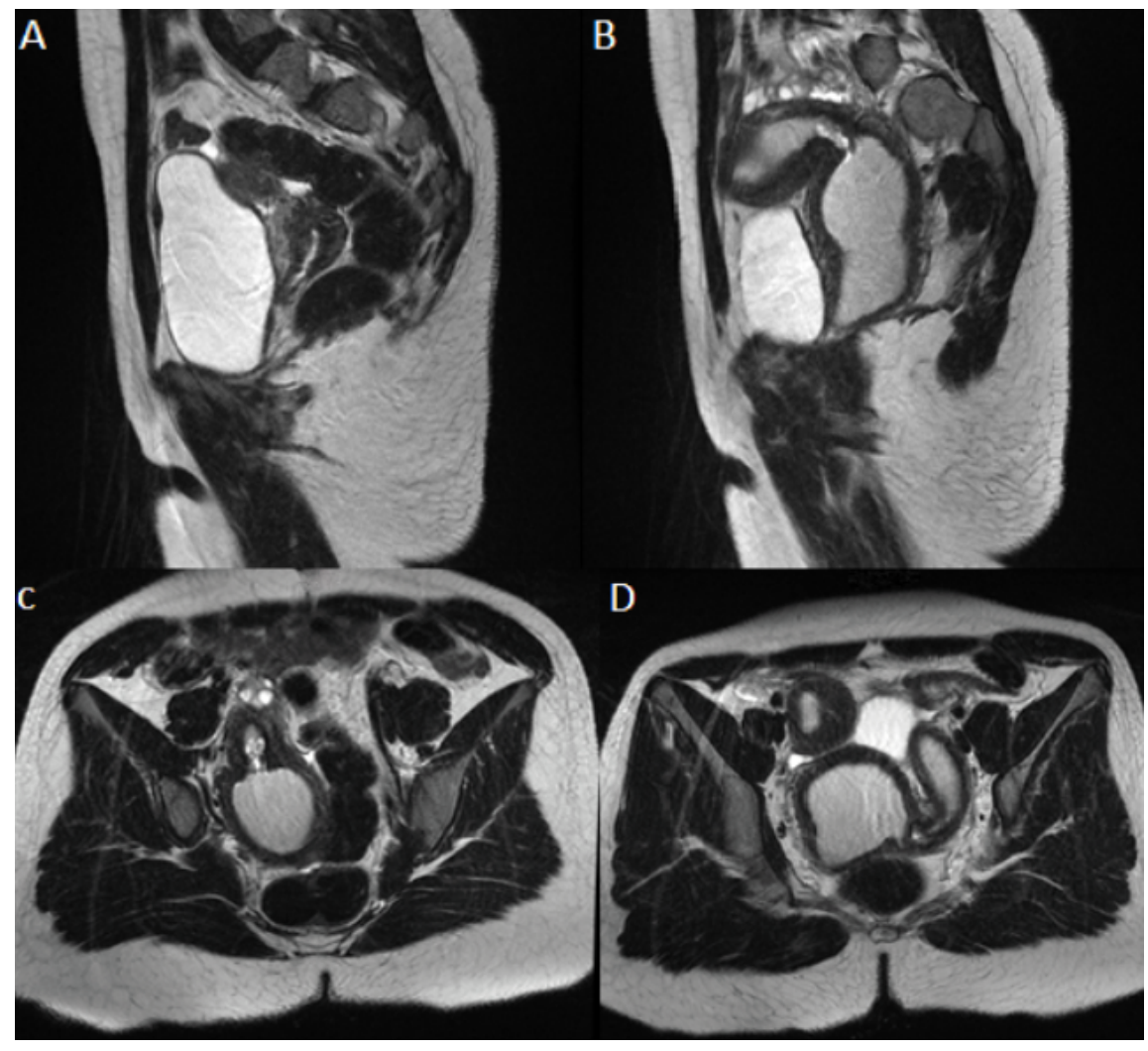

Figure caption list:

Figure1: Pelvic ultrasound image showing hematocolpos (echogenic contents).

Figure 2: A transabdominal ultrasound image showing the right dilated uterus with hematometra and the hematocolpos.

Figure 3: A )Sagittal magnetic resonance image (MRI) showing the normal uterine left cavity.B )Sagittal image of the same patient showing the dilated right uterine cavity and the hematocolpos.C )Coronal MRI showing the right cervix and the hematocolpos $\mathbf{D}$ )Coronal image showing the right uterine horn and the normal left uterine cavity with hematometra. 